(C) 2013 IEEE. Personal use of this material is permitted. Permission from IEEE must be obtained for all other uses, in any current or future media, including reprinting/republishing this material for advertising or promotional purposes,creating new collective works, for resale or redistribution to servers or lists, or reuse of any copyrighted component of this work in other works. DOI 10.1109/NBiS.2013.26

\title{
A Fuzzy-based System for Peer Reliability in JXTA-Overlay P2P Considering Number of Interactions
}

\author{
${\text { Evjola Spaho*, Shinji Sakamoto* }{ }^{*} \text { Leonard Barolli }{ }^{\dagger}, \text { Fatos Xhafa }^{\ddagger}, \text { Valbona Barolli }^{\S} \text { and Jiro Iwashige }}^{\dagger}$ \\ ${ }^{*}$ Graduate School of Engineering, \\ Fukuoka Institute of Technology (FIT), \\ 3-30-1 Wajiro-Higashi, Higashi-Ku, Fukuoka 811-0295, Japan \\ Email: evjolaspaho@hotmail.com,shinji.t.sakamoto@gmail.com \\ ${ }^{\dagger}$ Department of Information and Communication Engineering, \\ Fukuoka Institute of Technology (FIT), \\ 3-30-1 Wajiro-Higashi, Higashi-Ku, Fukuoka 811-0295, Japan \\ Email: barolli@fit.ac.jp,iwashige@fit.ac.jp \\ ${ }^{\ddagger}$ Department of Languages and Informatics Systems, \\ Technical University of Catalonia, \\ C/Jordi Girona 1-3, 08034 Barcelona, Spain \\ Email: fatos@lsi.upc.edu \\ $\S$ Department of Advanced Science \\ Hosei University \\ 3-7-2 Kajino-machi, Koganei-shi, Tokyo 184-8584, Japan \\ Email:valbona.barolli@gmail.com
}

\begin{abstract}
The trustworthiness of peers is very important for safe communication in P2P system. In this paper, we propose a fuzzy-based reliability system for P2P Communications in JXTA-Overlay. We use three input parameters: Number of Interactions (NOI), Amount of Data Exchanged $(A D E)$, and Reputation $(R)$ and the output is Peer Reliability $(P R)$. We evaluate the proposed system by computer simulations. The simulation results show that the proposed system have a good behaviour can be used successfully to evaluate the scale of reliability of the new peer connected in JXTA-Overlay.
\end{abstract}

Keywords-P2P, Fuzzy System, JXTA-Overlay, reliability.

\section{INTRODUCTION}

$\mathrm{P} 2 \mathrm{P}$ architectures will be very important for future distributed systems and applications. In such systems, the computational burden of the system can be distributed to peer nodes of the system. Therefore, in decentralized systems users become themselves actors by sharing, contributing and controlling the resources of the system. This characteristic makes P2P systems very interesting for the development of decentralized applications [1], [2].

In [1], it is proposed a JXTA-based P2P system. JXTAOverlay is a middleware built on top of the JXTA specification, which defines a set of protocols that standardize how different devices may communicate and collaborate among them. It abstracts a new layer on the top of JXTA through a set of primitive operations and services that are commonly used in JXTA-based applications and provides a set of primitives that can be used by other applications, which will be built on top of the overlay, with complete independence. JXTA-Overlay provides a set of basic functionalities, primitives, intended to be as complete as possible to satisfy the needs of most JXTA-based applications.

In P2P systems, each peer has to obtain information of other peers and propagate the information to other peers through neighboring peers. Thus, it is important for each peer to have some number of neighbor peers. Moreover, it is more significant to discuss if each peer has reliable neighbor peers. In reality, each peer might be faulty or might send obsolete, even incorrect information to the other peers. If a peer is faulty, other peers which receive incorrect information on the faulty peer might reach a wrong decision. Therefore, it is critical to discuss how a peer can trust each of its neighbor peers [3], [4].

The trustworthiness of peers is very important for safe communication in $\mathrm{P} 2 \mathrm{P}$ system. The trustworthiness of a peer can be evaluated based on the reputation and actual behavior of peers to provide services to other peers. However, in order to decide the peer trustworthiness are needed many parameters, which make the problem NP-hard.

Fuzzy Logic (FL) is the logic underlying modes of reasoning which are approximate rather then exact. The importance of FL derives from the fact that most modes of 


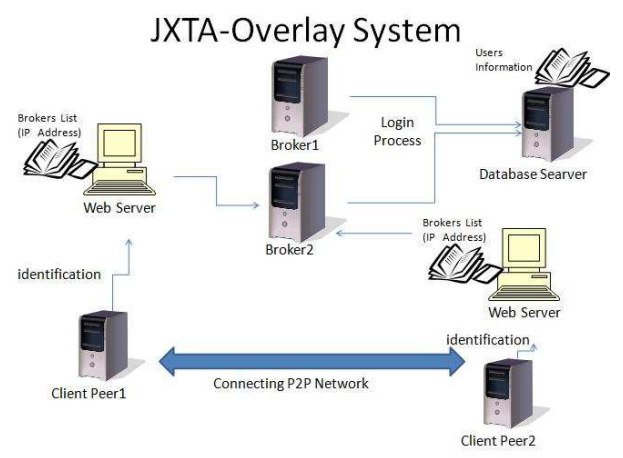

Figure 1: Structure of JXTA-Overlay system.

human reasoning and especially common sense reasoning are approximate in nature. FL uses linguistic variables to describe the control parameters. By using relatively simple linguistic expressions it is possible to describe and grasp very complex problems. A very important property of the linguistic variables is the capability of describing imprecise parameters.

The fuzzy set theory uses the membership function to encode a preference among the possible interpretations of the corresponding label. A fuzzy set can be defined by examplification, ranking elements according to their typicality with respect to the concept underlying the fuzzy set [5].

In this paper, we present a fuzzy-based peer reliability systems for JXTA-Overlay P2P platform considering Number of Interactions (NOI), Amount of Data Exchanged $(A D E)$, and Reputation $(R)$.

The structure of this paper is as follows. In Section II, we introduce JXTA technology and JXTA-Overlay. In Section III, we introduce FL used for control. In Section IV, we present our fuzzy-based peer reliability system. In section $\mathrm{V}$, we show the simulation results. Finally, conclusions and future work are given in Section VI.

\section{JXTA TECHNOLOGY AND JXTA-OVERLAY}

\section{A. JXTA Technology}

JXTA technology is a generalized group of protocols that allow different devices to communicate and collaborate among them. JXTA offers a platform covering basic needs in developing P2P networks [13].

By using the JXTA framework, it is possible that a peer in a private network can be connected to a peer in the Internet by overcoming existing firewalls. In this figure, the most important entity is the router peer. A router peer is any peer which supports the peer endpoint protocol and routing messages between peer in the JXTA networks. The procedure to overcome the firewall is as follows.

- In the Router Peer is stored the private address of Peer1 by using the HTTP protocol to pass the firewall from Peer1.

- The Router Peer receives the data from Peer2 and access the Private address of Peer1 to transmit the data.

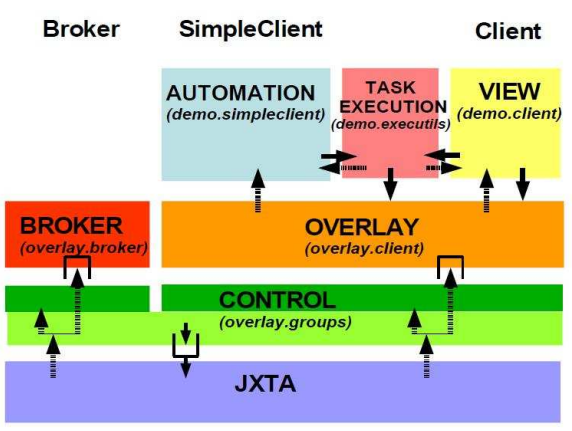

Figure 2: Internal Architecture of JXTA-Overlay.

JXTA is an interesting alternative for developing P2P systems and groupware tools to support online teams of students in virtual campuses. In particular, it is appropriate for file sharing given that the protocols allow to develop either pure or mixed $\mathrm{P} 2 \mathrm{P}$ networks. This last property is certainly important since pure $\mathrm{P} 2 \mathrm{P}$ systems need not the presence of a server for managing the network.

\section{B. JXTA-Overlay}

JXTA-Overlay project is an effort to use JXTA technology for building an overlay on top of JXTA offering a set of basic primitives (functionalities) that are most commonly needed in JXTA-based applications [14]-[16]. The proposed overlay comprises the following primitives:

- peer discovery,

- peer's resources discovery,

- resource allocation,

- task submission and execution,

- file/data sharing, discovery and transmission,

- instant communication,

- peer group functionalities (groups, rooms etc.),

- monitoring of peers, groups and tasks.

This set of basic functionalities is intended to be as complete as possible to satisfy the needs of JXTA-based applications. The overlay is built on top of JXTA layer and provides a set of primitives that can be used by other applications, which on their hand, will be built on top of the overlay, with complete independence. The JXTAOverlay project has been developed using the ver-2.3 JXTA libraries. In fact, the project offers several improvements of the original JXTA protocols/services in order to increase the reliability of JXTA-based distributed applications and to support group management and file sharing.

The architecture of the $\mathrm{P} 2 \mathrm{P}$ distributed platform we have developed using JXTA technology has two main peers: Broker and Client. Altogether these two peers form a new overlay on top of JXTA. The structrure of JXTA-Overlay system is shown in Fig 1.

\section{Internal Architecture of JXTA-Overlay}

Except Broker and Client peers, the JXTA-Overlay has also SimpleClient peers as shown in Fig. 2. The control 
layer interacts with the JXTA layer, and is divided into two parts: a lower part with functionality common to any kind of peer, and a higher part with functionality specific to Brokers and Clients.

- The common part provides functionality for doing JXTA messaging, discovery and advertisement.

- The Broker specific part provides functionality for managing groups of Brokers and keeping broker statistics.

- The Client specific part provides functionality for managing groups of Clients, keeping client statistics, managing its shareable files, managing the user configuration and creating the connection with a Broker.

The lower part enqueues the JXTA messages to be sent. Whenever a message arrives, the JXTA layer fires an event to the lower layer, which in turn fires a notifications to the upper layers.

\section{ApPlicAtion OF FUZZY LOGIC FOR CONTROL}

The ability of fuzzy sets and possibility theory to model gradual properties or soft constraints whose satisfaction is matter of degree, as well as information pervaded with imprecision and uncertainty, makes them useful in a great variety of applications.

The most popular area of application is Fuzzy Control (FC), since the appearance, especially in Japan, of industrial applications in domestic appliances, process control, and automotive systems, among many other fields.

\section{A. FC}

In the FC systems, expert knowledge is encoded in the form of fuzzy rules, which describe recommended actions for different classes of situations represented by fuzzy sets.

In fact, any kind of control law can be modeled by the FC methodology, provided that this law is expressible in terms of "if ... then ..." rules, just like in the case of expert systems. However, FL diverges from the standard expert system approach by providing an interpolation mechanism from several rules. In the contents of complex processes, it may turn out to be more practical to get knowledge from an expert operator than to calculate an optimal control, due to modeling costs or because a model is out of reach.

\section{B. Linguistic Variables}

A concept that plays a central role in the application of FL is that of a linguistic variable. The linguistic variables may be viewed as a form of data compression. One linguistic variable may represent many numerical variables. It is suggestive to refer to this form of data compression as granulation [6].

The same effect can be achieved by conventional quantization, but in the case of quantization, the values are intervals, whereas in the case of granulation the values are overlapping fuzzy sets. The advantages of granulation over quantization are as follows:
- it is more general;

- it mimics the way in which humans interpret linguistic values;

- the transition from one linguistic value to a contiguous linguistic value is gradual rather than abrupt, resulting in continuity and robustness.

\section{FC Rules}

FC describes the algorithm for process control as a fuzzy relation between information about the conditions of the process to be controlled, $\mathrm{x}$ and $\mathrm{y}$, and the output for the process $z$. The control algorithm is given in "if-then" expression, such as:

\section{If $\mathrm{x}$ is small and $\mathrm{y}$ is big, then $\mathrm{z}$ is medium;}

If $\mathrm{x}$ is big and $\mathrm{y}$ is medium, then $\mathrm{z}$ is big.

These rules are called $F C$ rules. The "if" clause of the rules is called the antecedent and the "then" clause is called consequent. In general, variables $x$ and $y$ are called the input and $z$ the output. The "small" and "big" are fuzzy values for $\mathrm{x}$ and $\mathrm{y}$, and they are expressed by fuzzy sets.

Fuzzy controllers are constructed of groups of these FC rules, and when an actual input is given, the output is calculated by means of fuzzy inference.

\section{Control Knowledge Base}

There are two main tasks in designing the control knowledge base. First, a set of linguistic variables must be selected which describe the values of the main control parameters of the process. Both the input and output parameters must be linguistically defined in this stage using proper term sets. The selection of the level of granularity of a term set for an input variable or an output variable plays an important role in the smoothness of control. Second, a control knowledge base must be developed which uses the above linguistic description of the input and output parameters. Four methods [7]-[10] have been suggested for doing this:

- expert's experience and knowledge;

- modelling the operator's control action;

- modelling a process;

- self organization.

Among the above methods, the first one is the most widely used. In the modeling of the human expert operator's knowledge, fuzzy rules of the form "If Error is small and Change-in-error is small then the Force is small" have been used in several studies [11], [12]. This method is effective when expert human operators can express the heuristics or the knowledge that they use in controlling a process in terms of rules of the above form.

\section{E. Defuzzification Methods}

The defuzzification operation produces a non-FC action that best represent the membership function of an inferred 


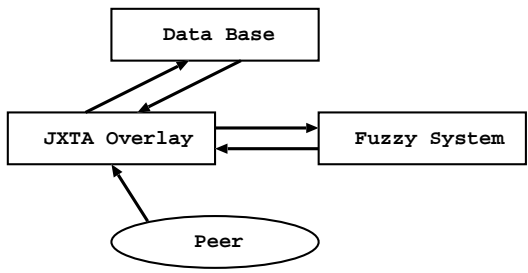

Figure 3: Peer reliability system for JXTA-Overlay.
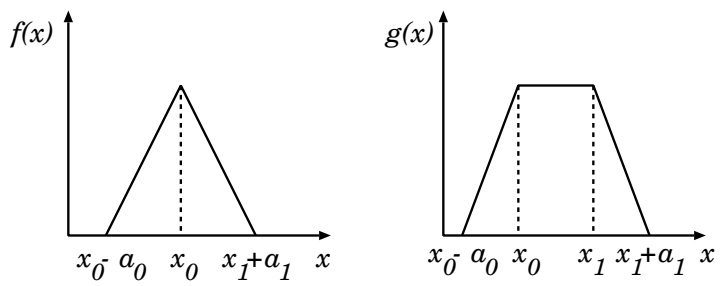

Figure 4: Triangular and trapezoidal membership functions.

FC action. Several defuzzification methods have been suggested in literature. Among them, four methods which have been applied most often are:

- Tsukamoto's Defuzzification Method;

- The Center of Area (COA) Method;

- The Mean of Maximum (MOM) Method;

- Defuzzification when Output of Rules are Function of Their Inputs.

\section{Proposed Fuzzy-Based Peer Reliability SYSTEM}

To complete a certain task in JXTA-Overlay network, peers often have to interact with unknown peers. Thus, it is important that peers must select reliable peers to interact. The number of interactions that a peer has with other peers in JXTA-Overlay P2P network is a very important factor that affects the peer reliability. Another important parameter that is connected with peer reliability is the amount of data exchanged. Selfish peers that benefits from the system without contributing any resources to the network have a low reliability. We use the past experiences of the peers that are saved in the database to select the most reliable candidate peer. Reputation is a peer's belief in another peer's capability based on recommendations received from other peers. Having reliable reputation information about the peers, our system can guide peers in their decision making to find other peers with good reputation.

Every time a peer joins JXTA-Overlay, parameters are fuzzified using fuzzy system, and based on the decision of fuzzy system a reliable peer is selected. After peer selection, the data for this peer are saved in the database as shown in Fig. 3.

In our proposed system, we use three input parameters: Number of Interactions (NOI), Amount of Data Exchanged $(A D E)$, and Reputation $(R)$ and the output is Peer Reliability
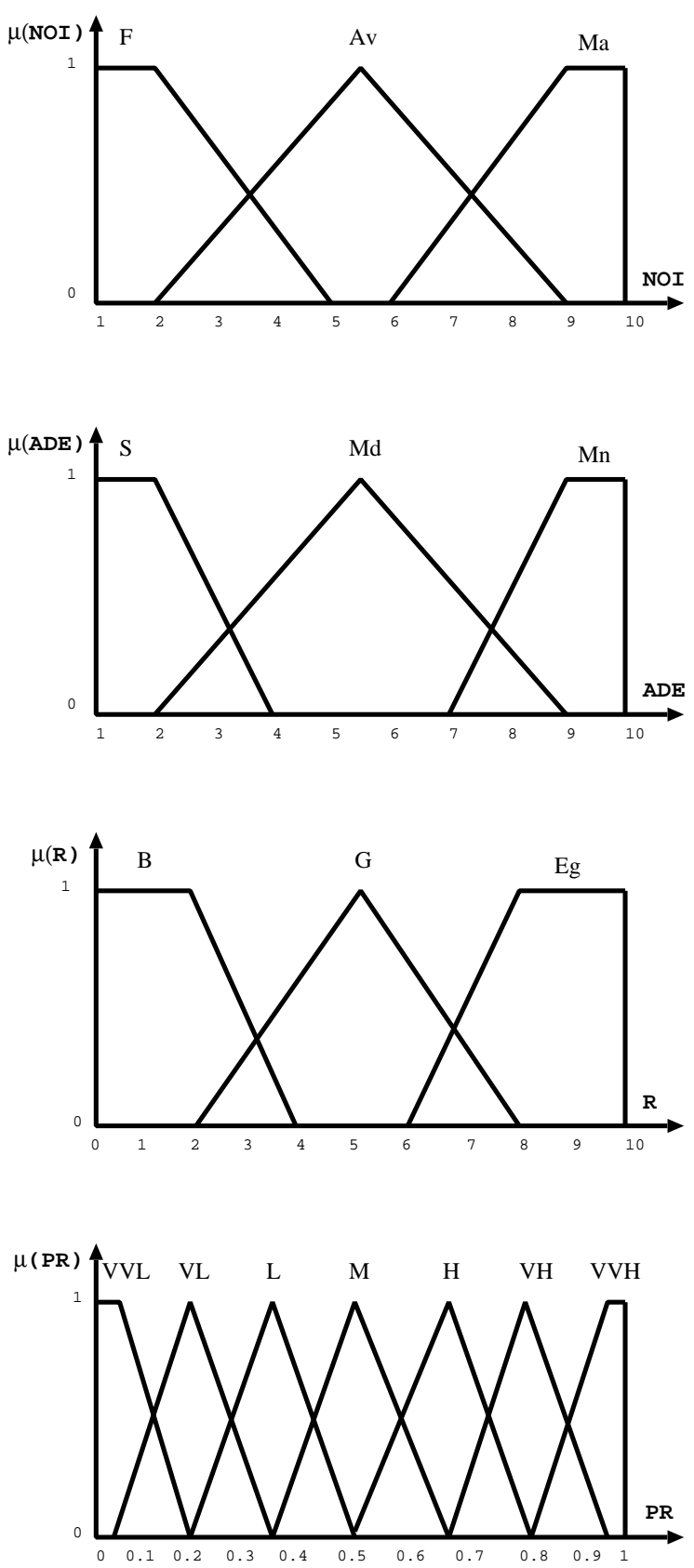

Figure 5: Membership functions.

$(P R)$. The term sets of NOI, ADE, and $R$ are defined respectively as:

$$
\begin{aligned}
\mu(N O I) & =\{\text { Few, Average, Many }\} \\
& =\{F, \text { Av, Ma }\} ; \\
\mu(A D E) & =\{\text { Small, Medium, Many }\} \\
& =\{S, M d, \text { Mn }\} ; \\
\mu(R) & =\{\text { Bad, Good, Extremely good }\} \\
& =\{B, G, E g\} .
\end{aligned}
$$


Table I: FRB.

\begin{tabular}{|c|c|c|c|c|}
\hline Rule & NOI & ADE & R & PR \\
\hline \hline 1 & F & S & B & VVL \\
\hline 2 & F & S & G & VVL \\
\hline 3 & F & S & Eg & L \\
\hline 4 & F & Md & B & VL \\
\hline 5 & F & Md & G & L \\
\hline 6 & F & Md & Eg & M \\
\hline 7 & F & Mn & B & L \\
\hline 8 & F & Mn & G & M \\
\hline 9 & F & Mn & Eg & H \\
\hline 10 & Av & S & B & VVL \\
\hline 11 & Av & S & G & L \\
\hline 12 & Av & S & Eg & M \\
\hline 13 & Av & Md & B & L \\
\hline 14 & Av & Md & G & M \\
\hline 15 & Av & Md & Eg & H \\
\hline 16 & Av & Mn & B & M \\
\hline 17 & Av & Mn & G & H \\
\hline 18 & Av & Mn & Eg & VH \\
\hline 19 & Ma & S & B & VL \\
\hline 20 & Ma & S & G & L \\
\hline 21 & Ma & S & Eg & M \\
\hline 22 & Ma & Md & B & M \\
\hline 23 & Ma & Md & G & H \\
\hline 24 & Ma & Md & Eg & VH \\
\hline 25 & Ma & Mn & B & H \\
\hline 26 & Ma & Mn & G & VVH \\
\hline 27 & Ma & Mn & Eg & VVH \\
\hline & & & & \\
\hline
\end{tabular}

and the term set for the output $(P R)$ is defined as:

$$
\begin{aligned}
\mu(P R)= & \{\text { Very Very Low, Very Low, Low, Middle, High } \\
& \text { Very High, Very Very High }\} \\
= & \{V V L, V L, L, M, H, V H, V V H\}
\end{aligned}
$$

As shown in Fig. 4, as membership functions we use triangular and trapezoidal membership functions. The $x_{0}$ in $f(x)$ is the center of triangular function, $x_{0}\left(x_{1}\right)$ in $g(x)$ is the left (right) edge of trapezoidal function, and $a_{0}\left(a_{1}\right)$ is the left (right) width of the triangular or trapezoidal function.

The membership functions for this system are shown in Fig. 5. In Table I, we show the Fuzzy Rule Base (FRB) of our system, which consists of 27 rules.

\section{Simulation Results}

In this section, we present the simulation results for our proposed system. In our system, we decided the number of term sets by carrying out many simulations in MATLAB. In Fig. 6, Fig. 7 and Fig. 8 are shown the simulation results of PR vs. R for different values of NOI and ADE. In Fig. 6 NOI is constant (1 unit) and ADE and R are increased. In Fig. 8, we show the simulation results NOI=10 units. In Fig. 7 we increase the NOI to 5 units. Also in this case NOI is constant while ADE and $\mathrm{R}$ are increased. In this three figures, we can distinguish three zones:

- Zone I, for R from 0 to 4 units,

- Zone II, for R from 4 to 6 units,

- Zone III, for R from 6 to 10 units.

In Zone I, when R is increased, PR is almost constant, but after it is increased for all values of ADE. PR increases

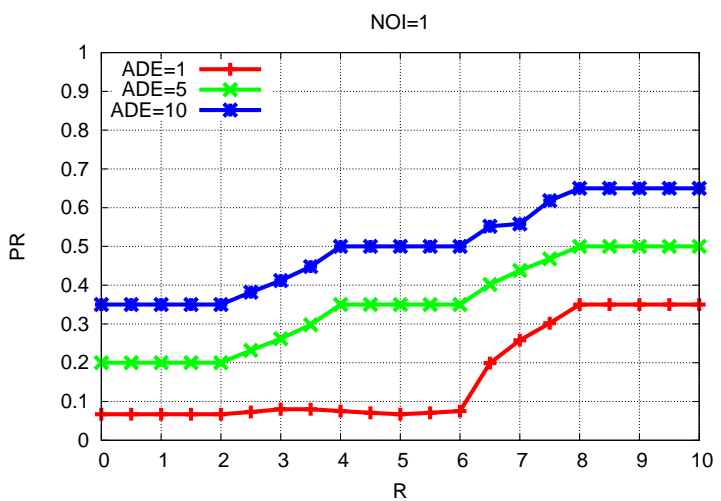

Figure 6: Simulation results for NOI=1.

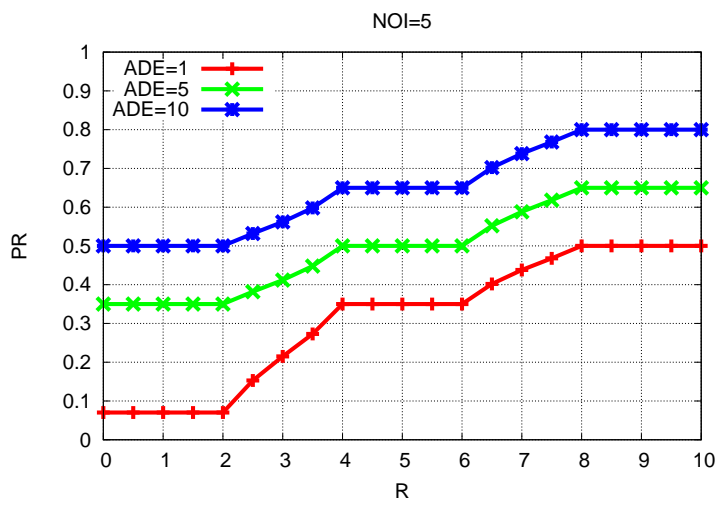

Figure 7: Simulation results for NOI=5.

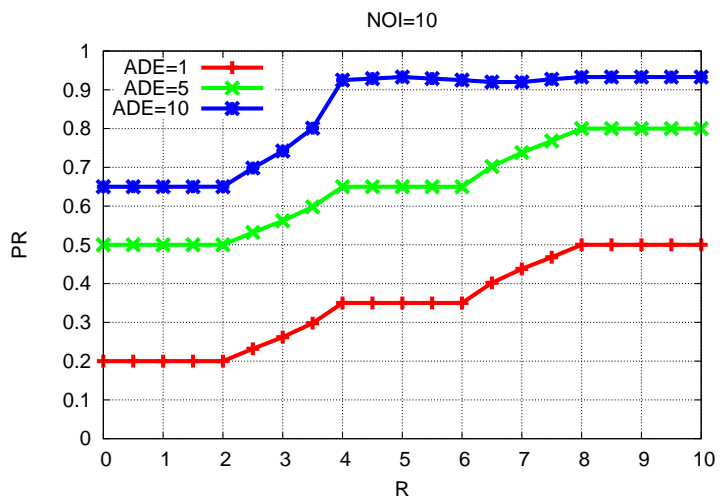

Figure 8: Simulation results for NOI=10.

with the increase of NOI. In Zone II, PR is increased when NOI and ADE between peers is increased. In Zone III, PR is almost constant, but it is increased with the increasing of $\mathrm{ADE}$ and NOI.

\section{CONCLUSions And Future Work}

In this work, we proposed a fuzzy-based peer reliability systems for JXTA-Overlay P2P system. We considered as input parameters: NOI, ADE and R and as output PR. We evaluate the performance of our proposed fuzzy system by computer simulations. From our MATLAB simulations, we 
found the following results.

- When the amount of exchanged date is small, the selfish peer benefits from the system without contributing any resources to the network and the PR is very low.

- With the increasing of R, ADE and NOI, PR is increased.

- Peer reliability is high when the peer have a good reputation and interacts with other peers exchanging its resources.

The proposed system have a good behavior and can be used successfully to evaluate the reliability of the new peer connected in JXTA-Overlay. In the future, we would like to consider other parameters and make extensive simulations to evaluate the proposed system.

\section{ACKNOWLEDGEMENTS}

This work is supported by a Grant-in-Aid for Scientific Research from Japanese Society for the Promotion of Science (JSPS). The authors would like to thank JSPS for the financial support.

\section{REFERENCES}

[1] F. Xhafa, R. Fernandez, T. Daradoumis, L. Barolli, S. Caballe, "Improvement of JXTA Protocols for Supporting Reliable Distributed Applications in P2P Systems", Proc. of NBiS-2007 (Regensburg, Germany), LNCS 4658, pp.345354, September 2007.

[2] L. Barolli, F. Xhafa, A. Durresi, G. De Marco, "M3PS: A JXTA-based Multi-platform P2P System and Its Web Application Tools", International Journal of Web Information Systems, Vol. 2, No. 3/4, pp. 187-196, 2006.

[3] A. Aikebaier, T. Enokido, M. Takizawa, "Reliable Message Broadcast Schemes in Distributed Agreement Protocols", Proc. of BWCCA-2010 (Fukuoka, Japan), pp. 242-249, November 2010.
[4] K. Watanabe, Y. Nakajima, T. Enokido, M. Takizawa, "Ranking Factors in Peer-to-Peer Overlay Networks", ACM Transactions on Autonomuous and Adaptive Systems (TAAS), Vol 2, No. 3, 11, pp. 1-26, September 2007.

[5] T. Terano, K. Asai, and M. Sugeno, "Fuzzy Systems Theory And Its Applications", Academic Press, INC. Harcourt Brace Jovanovich, Publishers, 1992.

[6] A. Kandel, "Fuzzy Expert Systems", CRC Press, 1992.

[7] H. J. Zimmermann, "Fuzzy Set Theory and Its Applications", Kluwer Academic Publishers, Second Revised Edition, 1991.

[8] F. M. McNeill, and E. Thro, "Fuzzy Logic. A Practical Approach”, Academic Press, Inc., 1994.

[9] L. A. Zadeh, J. Kacprzyk, "Fuzzy Logic For The Management of Uncertainty", John Wiley \& Sons, Inc., 1992.

[10] T. J. Procyk and E. H. Mamdani, "A Linguistic Selforganizing Process Controller", Automatica, Vol. 15, No. 1, pp. 15-30, 1979.

[11] G. J. Klir, and T. A. Folger, "Fuzzy Sets, Uncertainty, And Information", Prentice Hall, Englewood Cliffs, 1988.

[12] T. Munakata, and Y. Jani, "Fuzzy Systems: An Overview", Commun. of ACM, Vol. 37, No. 3, pp. 69-76, March 1994.

[13] D. Brookshier, D. Govoni, N. Krishnan, J.C Soto, "JXTA: Java P2P Programming", Sams Publishing, 2002.

[14] K. Matsuo, L. Barolli, V. Kolici, F. Xhafa, A. Koyama, A. Durresi, "Stimulation Effects of SmartBox for E-learning Using JXTA-Overlay P2P System", Proc. of CISIS-2009, pp. 231-238, 2009.

[15] Y. Ogata, K. Matsuo, E. Spaho, L. Barolli, F. Xhafa, "Implementation of SmartBox End-Device for a P2P System and Its Evaluation for E-Learning and Medical Applications", Proc. of BWCCA-2010, pp. 794-799, 2010.

[16] E. Spaho, K. Matsuo, L. Barolli, F. Xhafa, J. Arnedo-Moreno, V. Kolici, "Application of JXTA-Overlay Platform for Secure Robot Control", Journal of Mobile Multimedia, Vol. 6, No. 3, pp. 227-242, 2010. 